\title{
Fitting Measured Emittance Data
}

Philip F. Meads, Jr.

7053 Shirley Drive

Oakland, California 94611-1631

\section{Abstract}

Emittance measurements have been made at several points in the 50$\mathrm{MeV}$ Neutral Particle Beam Test Stand of ANL. These measurements were made with segmented Faraday cups that measure the angular distribution after passing the beam through a movable slit. The measured distribution is very nonuniform, and thus the Twiss parameters for the best fit at a given emittance depend on the ellipse area. We here describe a program that optimizes the Twiss parameters and the ellipse center as a function of the emittance. This is done separately in the two lateral phase planes. The $\beta$ and $\alpha$ parameters and the ellipse center are adjusted such that the total count contained within the ellipse is maximized. The optimization method is due to Powell and Brent; it does not require the evaluation of derivatives. Input data are read as a two-dimension array (displacement and angle). For cells containing the ellipse, the incremental count used is estimated by determining the intercepts of the ellipse in the two dimensions and approximating that portion of the curve by a straight line. Optional initial smoothing of the measured data is provided. This analysis method differs from that in the paper by Rosing, Hummer, and Zolecki (this conference) where they find a set of Twiss parameters, the center of the beam and emittance that fits an assumed distribution.

\section{Introduction}

As part of designing the transport line for the $50 \mathrm{MeV}$ Neutral Particle Beam Test Stand, we sought to determine the characteristics of the beam exiting the ANL linac. This linac has been running for many years as the injector to the ANL rapid cycling synchrotron, and little analysis of the beam had been done in recent times. The program required a beam collimated to a very small emittance but with as large a current as possible. Because this beam, as the case for most beams, has a nonuniform brightness distribution, the optimum matching criteria depend on the emittance to be accommodated. So, given an emittance, we wished to determine the optimum centering for the beam and the values of the Twiss parameters, $\alpha$ and $\beta$ that maximize the current contained within that emittance. With this approach, the matching changes with changes in the emittance.

The complementary approach, described by Hummer and Zolecki ${ }^{1}$, looks for a best fit for the Twiss parameters and the center, demanding that these not depend on the emittance. This approach has the attraction that the matching requirements do not change over the range of emittances that are to be passed by the system, but this convenience comes at the cost of accepting less than the maximum current possible.

Wadlinger suggested a method of determining the minimum volume hyperellipsoid that just contains an observed particle distribution. ${ }^{2}$ Keller, Sherman and Allison have treated the problem of determining the minimum area ellipse that just contains the two dimensional particle distribution. ${ }^{3}$ Many others have dealt with this problem with as many different approaches.

\section{Method}

Measurements were made at several points in the ANL beam line, using a segmented Faraday cup array. This array is comprised of a stack of insulated $1 \mathrm{~mm}$ thick copper plates whose current can be monitored. A programmable slit ahead of this array selects one value of displacement that will be passed through to the segmented Faraday cup array. The distribution of intensity with respect to angle is then obtained from the segmented Faraday cups. The measurements are repeated for a number of locations for the programmable slit. The array can be rotate to measure either the horizontal or the vertical plane at any one time. We thus obtain a two dimensional array of intensities to which we now wish to fit emittance ellipses. The first index represents different slit positions, whereas the second represents the different Faraday cups. As the slit is shifted, the zero slope ray is shifted to adjacent Faraday cups; thus the data must be modified to yield intensity as a function of displacement and angle.

The measured intensity array is loaded and shifted so each row corresponds to one angle and each column to one displacement. An option is provided at this point to compare each cell to its four neighbors and to smooth the distribution, but this does spread the distribution.

A series of ellipses is generated for the current emittance. An ellipse is specified by $\beta, \alpha, x_{0}$, and $x_{0}^{\circ}$ (center). The first task is to go through the entire bin array and to decide for each comer whether it is within the ellipse or not. This being a Pascal code, the results are stored in the Boolean array InEllipse[ix,iy]. Next we look for all bin boundaries that are cut by the ellipse by looking for adjacent corners where one is in the ellipse and the other not. For each such boundary, we calculate and store the intercept, measured from the lower left comer in the array pair xint [ix,iy] (where the ellipse cuts a horizontal line) and yint[ix,iy] (where the ellipse cuts a vertical line).

Now we accumulate the total number of counts in the ellipse as follows. If all four comers of a bin are in the ellipse, we clearly add the measured current stored in that bin. If none of the corners are in the ellipse, the contents of that bin are ignored. If 1 to 3 comers are in the ellipse, then we calculate the fraction of the bin area that is in the ellipse by connecting the intercepts with straight lines. We add that fraction of the stored bin current. We do get continuous functions this way, but derivatives would be questionable.

The optimum values of the four ellipse parameters are determined by minimizing the negative of the total count, where the above procedure is used to return the value of the function being minimized. The routines used are the Pascal versions from the book, Numerical Recipes. The method used is that of Powell as modified by Brent; derivatives are not used. Initially line minimization is used along each of the four cardinal axes. Then, depending upon a test, one of the coordinates may be replaced by a new coordinate that is the new direction resulting from the four successive line minimizations. The choice of which coordinate to drop is important, because an inappropriate choice can result in the subsequent minimization being restricted to a subspace of the original four dimensional space. As implemented here, the one dropped is that that produced the biggest gain. This will be the closest to the new coordinate. The process is repeated: a series of four line minimizations along the current cardinal axes and the replacing of one of these axes by a new axis. The minimization algorithm is terminated when the changes being made become smaller than the square root of the precision desired.

Having found the optimum ellipse parameters for the given emittance, the emittance is reduced by an input factor, and the process is repeated.

'Work performed under the auspices of the US DOE and the US SDC.

U.S. Government work not protected by U.S. copyright. 


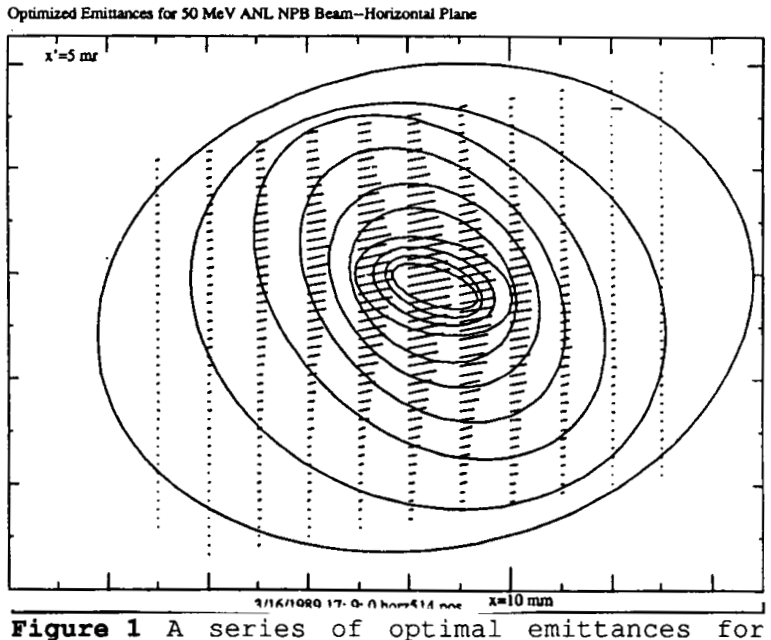
the ANL $50 \mathrm{MeV}$ beam.

Output is provided as a PostScript plot or a Tektronix 4014 plot with an accompanying table. The bin populations are shown on the plot as a series of slanted line segments whose length is proportional to the current stored in the bin. This yields a quasi three dimensional graph. On top of this, the series of ellipses is plotted. We show such a plot as Figure 1.

\section{References}

1. Hummer and Zolecki, The Measurement of the Twiss Parameters by Using SFCs, paper T54, this conference.

2. Wadlinger, General Least-Squares Fitting Procedures to Minimize the volume of a Hyperellipsoid, 1979 Linear Accelerator Colnference (BNL).

3 Keller, Sherman, Allison, Use of a Minimum-Ellipse Criterion in the Study of Ion-Beam Extraction Systems, IEEE Trans. Nucl. Sci, NS-32, p. 2579 (1985). 